\title{
Predictors and risk model development for menopausal age in fragile $X$ premutation carriers
}

\author{
Marian A. Spath, MD ${ }^{1,2}$, Ton B. Feuth, MSc ${ }^{3}$, Arie P. T. Smits, PhD ${ }^{2}$, Helger G. Yntema, PhD ${ }^{2}$, \\ Didi D. M. Braat, MD, PhD $D^{1}$, Chris M. G. Thomas, PhD ${ }^{1,4}$, Ad Geurts van Kessel, PhD ${ }^{2}$, \\ Stephanie L. Sherman, $P h D^{5}$, and Emily G. Allen, $P h D^{5}$
}

\begin{abstract}
Purpose: Women who carry a fragile $\mathrm{X}$ mental retardation 1 premutation are at risk for fragile $\mathrm{X}$-associated primary ovarian insufficiency and should be counseled for a potentially reduced fertility. Multiple factors can affect the age of onset and severity of fragile X-associated primary ovarian insufficiency. In this study, we assessed the predictive power of several factors with menopausal age, a surrogate measure of onset of fragile X-associated primary ovarian insufficiency. Methods: Genetic, environmental, and reproductive factors were analyzed by Cox proportional hazard models in 1068 women, 385 of fragile $\mathrm{X}$ families ascertained through the Nijmegen study and 683 of fragile $X$ families or general population families ascertained through the Atlanta study. Results: The highest association with menopausal age among fragile $\mathrm{X}$ mental retardation 1 premutation carriers was found for risk index by CGG repeat size (hazard ratio, 1.43) and smoking (hazard ratio, 1.34). Women from the Nijmegen cohort showed an overall lower age at menopause onset, for which a correction was made. A prediction model based on these two parameters, mean menopausal age of first-degree relatives with the same mutation status and the correction for ascertainment site, estimated the probability of becoming postmenopausal for premutation carriers, with a margin of $7-10 \%$. Conclusion: We conclude that this model serves as a first step toward clinical application of fragile X-associated primary ovarian insufficiency prediction. Genet Med 2011:13(7):643-650.
\end{abstract}

Key Words: FMRI premutation, menopause, fragile X-associated primary ovarian insufficiency (FXPOI), prediction

In the early 1990s, premature ovarian failure (POF), or cessation of menses before the age of 40 years, was noted among heterozygous carriers of the fragile $\mathrm{X}$ mental retardation 1 $(F M R l)$ premutation. ${ }^{1,2}$ Additional studies showed that the risk of POF was almost exclusively seen in females carrying a premutation in the FMR 1 gene and that approximately $20 \%$ of these carriers had POF. ${ }^{3,4}$ To better denote the complete spec-

From the Departments of ${ }^{1}$ Obstetrics and Gynecology, ${ }^{2}$ Human Genetics, ${ }^{3}$ Epidemiology, Biostatistics and HTA, and ${ }^{4}$ Laboratory Medicine, Radboud University Nijmegen Medical Centre, Nijmegen, The Netherlands; and ${ }^{5}$ Department of Human Genetics, Emory University, Atlanta, Georgia.

Marian A. Spath, MD, Department of Obstetrics and Gynecology (791), Radboud University Nijmegen Medical Centre, P.O. Box 9101, Nijmegen 6500 HB, The Netherlands. E-mail: M.Spath@obgyn.umcn.nl.

M.A.S. and T.B.F. contributed equally to this work.

Disclosure: The authors declare no conflict of interest.

Supplemental digital content is available for this article. Direct URL citations appear in the printed text and are provided in the HTML and PDF versions of this article on the journal's Web site (www.geneticsinmedicine.org).

Submitted for publication December 7, 2010.

Accepted for publication February 23, 2011.

Published online ahead of print May 18, 2011

DOI: $10.1097 /$ GIM.0b013e31821705e5 trum of this disorder, including altered menstrual cycles, altered hormone profiles, infertility, and intermittent ovarian function, this premutation disorder was renamed as fragile $\mathrm{X}$-associated primary ovarian insufficiency (FXPOI), ${ }^{5}$ defined as amenorrhea for at least 4 months and two serum follicle-stimulating hormone levels above $40 \mathrm{IU} / \mathrm{L}$.

The FMRI premutation represents an expansion of a trinucleotide (CGG) repeat in the $5^{\prime}$ untranslated region of the FMRI gene. ${ }^{6}$ Based on the number of repeats present and their instability when passed from one generation to the next, four types of FMR 1 alleles can be distinguished: (1) a normal allele with $<45$ repeats, which is stably inherited; (2) an intermediate mutant allele with 45-54 repeats, which may show instability on maternal or paternal transmission; (3) a premutation allele with 55-200 repeats, which can expand to a full mutation within one generation; and (4) a full mutation allele with at least 200 CGG repeats (American College of Medical Genetics practice guidelines; 2007), which results in an intellectual and developmental disability syndrome, the fragile $\mathrm{X}$ syndrome. ${ }^{7}$ Women who carry the premutation should be informed not only about their risk for having a child with fragile $\mathrm{X}$ syndrome but also about their potential risk for FXPOI, as symptoms include infertility, early cessation of menses, and early exposure to estrogen deficiency.

In the general population, menopause ranges from ages 40 to 60 years with a mean of 51 years $^{8}$ and seems to be modulated by both environmental and genetic factors. ${ }^{9-12}$ Allen et al. ${ }^{13}$ showed that smoking led to an earlier age at menopause among premutation carriers; however, the association of menopausal age with other factors, such as menarche and parity is still a matter of debate. Although both environmental and reproductive factors contribute to variance in age at menopause, genetic factors seem to act as superior predictors for age at menopause. ${ }^{12}$ The size of the FMR1 CGG repeat is a major risk factor for FXPOI. Within the FMR1 premutation range of 55-200 repeats, Allen et al. ${ }^{13}$ and Sullivan et al. ${ }^{14}$ identified high-risk alleles of 80-99 repeats. This nonlinear association was subsequently confirmed by others. ${ }^{15,16}$ The subdivision of the premutation ranges used by Allen et al. ${ }^{13}$ into low (55-79 repeats), medium (80-99 repeats), and high (100-200 repeats) has, however, been based on the risk to expand to a full mutation, ${ }^{17}$ and any detailed information on high-risk alleles for FXPOI is, therefore, still lacking. In this study, we have developed an integrative model that allows the prediction of the risk to develop FXPOI in individual premutation carriers. Such a model would serve as an excellent adjunct to current fertility counseling modalities and the clinical intervention in premutation carriers.

\section{METHODS}

\section{Study population}

The study cohort comprised participants ascertained through two centers, the Radboud University Nijmegen Medical Centre, Nijmegen, the Netherlands (Nijmegen cohort), and the Emory University Department of Human Genetics, Atlanta, Georgia (At- 


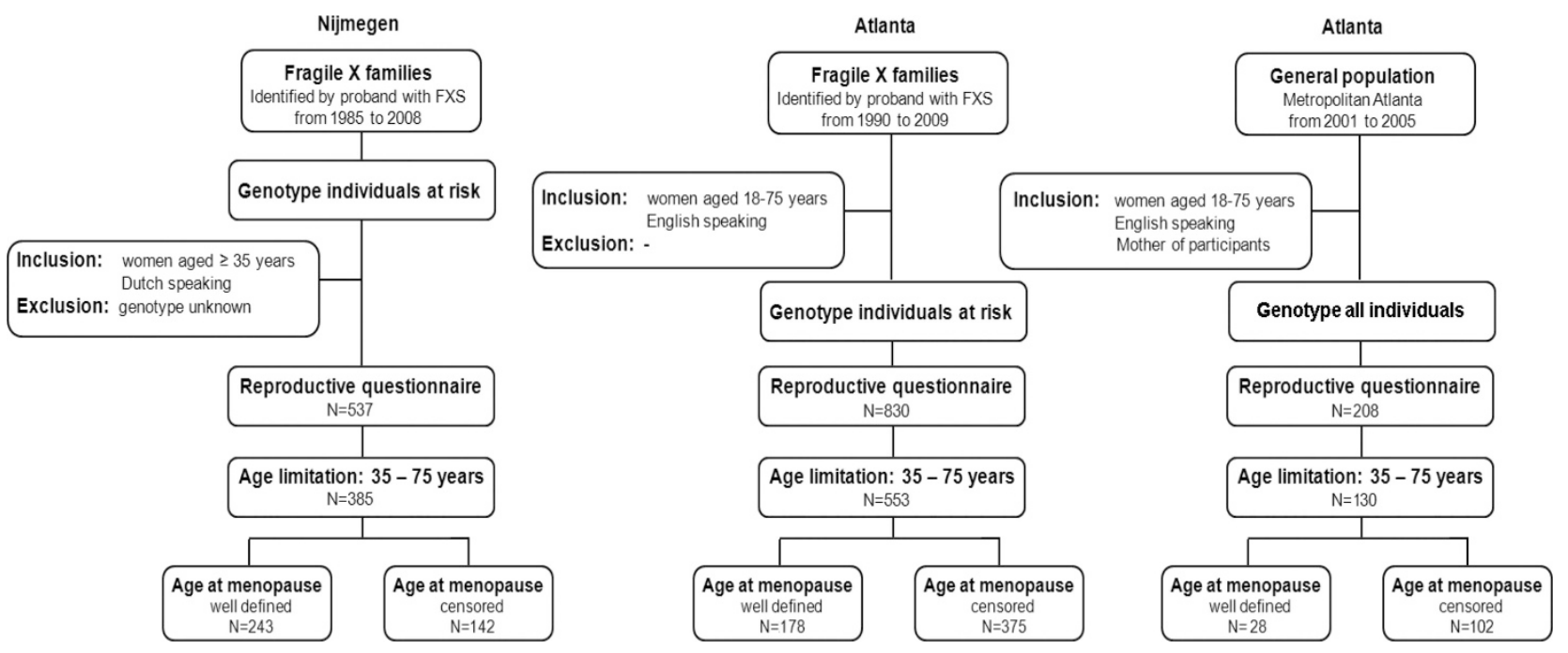

Fig. 1. Flowchart of the study population.

lanta cohort). The Dutch study was approved by the Institutional Review Board of the Radboud University Nijmegen Medical Centre. The Atlanta protocol and consent forms were approved by the Institutional Review Board at Emory University.

All fragile X families that were diagnosed at the Department of Human Genetics Nijmegen between 1984 and 2008 were ascertained for this study. All female relatives of a proband at age 35 years or older were approached to participate in this study and asked to complete a general health and reproductive history questionnaire. A detailed description of the study population is depicted in Figure 1. The Atlanta cohort comprised women who were recruited through the Emory Study of Adult Learning and Reproduction. Ascertainment protocols have been described in detail by Sullivan et al. ${ }^{14}$

\section{Data collection}

\section{Reproductive history questionnaire}

For both studies, comparable questionnaires on general health and reproduction were administered by phone, in written format, or through an internet survey. Both reproductive questionnaires gathered information on time since last menstrual period, cause for cessation of menstrual periods (e.g., menopause, hormones, and surgery), age at last period, age at start hormone use/hormone replacement therapy and oral contraceptives (OCs), time period of hormone use, and current hormone use.

\section{Definition of age at menopause}

Age at menopause was used as a surrogate for severity for FXPOI and was defined as amenorrhea for more than 1 year due to natural menopause. Ages at menopause for all women with other reasons than natural menopause for cessation of menstrual cycles were "censored." For women who had their last period due to surgery (e.g., hysterectomy or oophorectomy), chemotherapy, radiation or pregnancy, menopausal age was censored at age at last period. Women who still had menstrual cycles (last period $<1$ year) without current hormone use were censored at age at interview. Women with recent periods and current hormone use were censored at age when hormone use was started.

\section{FMR1 CGG repeat size measurement}

From the participants included in the Dutch study, DNA was extracted from peripheral blood cells. Polymerase chain reaction amplification of the FMRI alleles was performed using 6-carboxyfluorescein labeled reverse primers as described by $\mathrm{Fu}$ et al. ${ }^{18}$ Polymerase chain reaction conditions are available on request. Fragment lengths were measured using an ABI prism 3730 DNA Analyzer (Applied Biosystems, Foster City, CA) and quantified using ABI prism Genemapper Analysis Software v4.0 (Applied Biosystems) up to one or two repeats accurate until 130-140 repeats. From the women participating through Atlanta, DNA was extracted from buccal samples or peripheral blood cells using Qiagen QiAmp DNA Blood Mini Kit. FMR1 CGG repeat sizes were determined by a fluorescent-sequencer method as described by Sullivan et al., ${ }^{14}$ with the same accuracy as the Dutch method, up to 100 repeats and with an inaccuracy of 5-10 repeats for repeat sizes 100-140.

\section{Statistical analysis}

The data were analyzed using age at menopause, a surrogate for FXPOI, as outcome variable in Cox proportional hazards models. To this end, the time origin was set at the age of 25 years, and the time from 25 years to start menopause was considered as the time to event. To adjust for the correlation among outcomes within the same family, we applied robust estimates each time we made use of a Cox regression mode. ${ }^{19}$ Analyses were performed separately for FMRI premutation carriers and noncarriers.

\section{Construction of risk index for earlier menopause based on FMR1 CGG repeat size}

Cox regression with robust estimations were used to determine risk estimates for menopause at a younger age based on FMR1 CGG repeat size. A reference region based on most common repeat sizes encountered in the general population was defined to be $28-33$ repeats. ${ }^{18,20}$ For repeat sizes $20-28$ and 34-100 repeats, a set of moving windows with a width size of 11 repeats was created. In case of overlap between window and reference region, repeats present in the overlap were removed from the window. The menopausal age for subjects within a 
window was compared with menopausal age of subjects in the reference region, resulting in a window-specific hazard ratio (HR) where the index $r$ refers to the central repeat size in the window. To determine these HRs, we applied Cox regression with robust estimations. Because repeat size determination of $100-130$ repeats was less accurate for the Atlanta subset, a wider window ( \pm 10 repeats) around the reported repeat size was used. As sizes above 130 repeats could not be determined with accuracy for either cohort, they were not assessed in this hazard risk analysis. To reduce the noise in the resulting HR graph, we superimposed a smoothed curve that is fitted to the HRs by repeat scatter plot. To this end, we used the LOWESS smoother with smoothing factor $=0.1 .^{21}$ The resulting variable is referred to as the "Risk Index by Repeat size" (RIR) and ranged from 1 to 3.6 , with 1 indicating similar risk for menopause as women with repeat size $28-33$ and 3.6 indicating an odds of 3.6 of becoming postmenopausal per unit time compared with women with repeat sizes $28-33$.

\section{Assessment of putative predictors for menopausal age in premutation carriers}

Predictive factors for age at menopause were based on previous studies on menopause in general and included smoking, body mass index (BMI), age at menarche, OC use, and parity. ${ }^{12,22,23}$ The abundant evidence of heritability of menopausal age, and the large additive genetic component that Hunter et al. ${ }^{24}$ showed to be present after correction for the premutation repeat size, required a representation into our putative predictor assessment as well. To this end, for each subject, the mean age at menopause of first-degree relatives with the same mutation status (premutation if 55-200 repeats and noncarrier if $\leq 54$ repeats) was determined. If a subject was lacking a first-degree relative with the same mutation status and well-defined age at menopause, the median age at menopause observed in subjects (well-defined age at menopause) of the same premutation status, 44 years for carriers and 50 years for noncarriers, was employed. Because this study comprises participants from two separate studies from different centers, we also considered ascertainment location as a possible relevant explanatory variable. Putative predictors were assessed as continuous variables, except for smoking and $\mathrm{OC}$ use, which were dichotomized for ever smoking or ever OC use. As a first step in the modeling process, we assessed various potential predictors for menopausal age using univariate Cox models.

\section{Modeling process and validation}

For model building, backward selection was applied, starting with the set of variables that showed significance in the univariate analyses. For each potential predictor, the proportional hazard assumption was determined by inspecting plots of weighted Schoenfeld residuals against age at menopause. ${ }^{25}$ The appropriate functional form of continuous predictors was checked using martingale residuals as described in Ref. 26. To assess the fit of each resulting model, the subjects were divided into three tertile groups based on their values of the linear part of the Cox regression model. For each tertile, the Kaplan-Meier curve and the mean of the predicted Cox survival curves were computed. A perfect fit should give comparable curves for each tertile. Both resulting Cox models were validated using Efron's optimism bootstrap to estimate the optimism in $R^{2}$ and the concordance index $c$, to quantify the overfitting and to assess the calibration needed. ${ }^{27}$

\section{RESULTS}

\section{Delineation of the study populations}

In total, 1575 women were ascertained in the original studies, 537 from the Nijmegen cohort and 1038 from the Atlanta cohort. Approximately $90 \%$ of them were white. As the number of women from other ethnicities was too small to denote an effect, all non-whites were excluded from the initial cohort. To correct for age differences in recruitment schemes (Nijmegen recruited 35 years and older and Atlanta recruited $18-75$ years), we selected only women aged 35-75 years from both cohorts, resulting in a final study cohort of 1068 women. The characteristics of the final study cohort are listed in Table 1. Women

Table 1 Characteristics of the study population by mutation status

\begin{tabular}{|c|c|c|c|c|c|}
\hline & \multicolumn{2}{|c|}{ Premutation } & \multicolumn{2}{|c|}{ Noncarriers } & \multirow[b]{2}{*}{ Total } \\
\hline & Nijmegen & Atlanta & Nijmegen & Atlanta & \\
\hline Age at ascertainment (yr) & 56.4 & 49.6 & 54.6 & 52.7 & 53.0 \\
\hline \multicolumn{6}{|l|}{ Age at menopause (yr) } \\
\hline Well defined (\%) & $42.5(72)$ & $43.5(34)$ & $48.3(58)$ & $49.9(25)$ & $45.9(42)$ \\
\hline Censored $(\%)$ & $41.7(28)$ & $39.0(66)$ & $42.9(42)$ & $42.2(75)$ & $41.0(58)$ \\
\hline Menarche (yr) & 13.2 & 12.4 & 13.1 & 12.5 & 12.7 \\
\hline Parity & 2.2 & 2.1 & 2.0 & 2.1 & 2.1 \\
\hline Ever used hormones (\%) & $79 \%$ & $80 \%$ & $74 \%$ & $85 \%$ & $80 \%$ \\
\hline Ever smoking (\%) & $41 \%$ & $36 \%$ & $35 \%$ & $40 \%$ & $38 \%$ \\
\hline BMI & 24.6 & 27.0 & 25.8 & 27.2 & 26.5 \\
\hline \multicolumn{6}{|l|}{ Ascertainment $(N)$} \\
\hline Fragile X family, USA & & 370 & & 183 & $51.7 \%$ \\
\hline General population, USA & & 3 & & 127 & $12.2 \%$ \\
\hline Fragile X family, NL & 144 & & 241 & & $36.1 \%$ \\
\hline
\end{tabular}


included from Nijmegen were older at interview and, thus, had more often reached menopause $(72 \%)$ compared with women included from Atlanta, of whom 34\% had a well-defined age at menopause. In addition, women from the Nijmegen cohort exhibited an overall significant earlier menopausal age. When comparing the distribution of FMRI CGG repeat sizes of the Nijmegen and Atlanta cohorts, no difference was seen $(P=$ 0.58 , Mann-Whitney $U$ test)

\section{Definition of putative predictors}

\section{Risk index for earlier menopause based on FMR1 CGG repeat size}

To incorporate the nonlinear association of repeat size and risk for FXPOI, a risk index based on repeat size was developed. To explore possible differences between the two geographic ascertainment sites, unsmoothed results are presented separately. By doing so, both the Nijmegen and Atlanta cohorts exhibited no risk for earlier menopause for repeat sizes in the normal or intermediate range, as depicted by the $95 \%$ confidence interval $(\mathrm{CI})$ that includes $\mathrm{HR}=1$. In addition, both analyses showed that the risk for earlier menopause starts at 64 CGG repeats, at which the lower CI rises above a HR of 1 (Fig. 2, A and B). Also, some differences were seen between the two ascertainment sites, i.e., the HRs for 64 repeats or more were lower for the Nijmegen study cohort as for that from Atlanta. Also, within the Nijmegen cohort, the HRs were significantly elevated until repeat sizes of 90, whereas for the Atlanta cohort, the significantly elevated HRs reached 100 repeats. Although differences in HRs were seen, the shape of the curves was found to be similar, thus allowing pooling of both datasets. For the total study population, a significant high risk (lower confidence band $>1$ ) started above 62 repeats, then varied from 1.5 to 3.8 and, finally, became nonsignificant at 120 repeats (Fig. 2C). Repeat sizes of 131-200 $(n=21)$ had a HR of 2.1 (95\% CI: $1.0-4.3)$, with a significant isolated HR peak at 160 repeats (data not shown). The value of the smoothed LOWESS curve was used to assess a predictor to which we will refer to as the RIR.

\section{Value of putative predictors}

To estimate the value of all putative predictors, they were assessed for their association with age at menopause in univariate and multivariate Cox analyses with robust estimations in the subgroups of premutation carriers and noncarriers separately. For all potential predictors, no relevant violation of the proportional hazards model assumption was found, and all
A

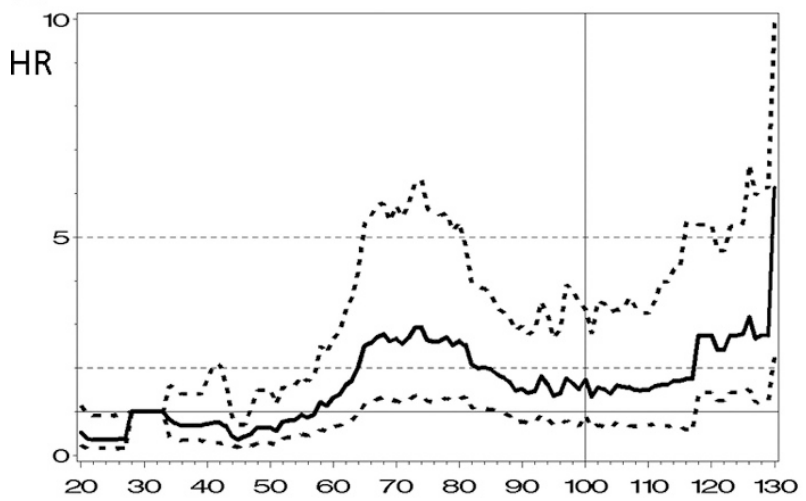

B

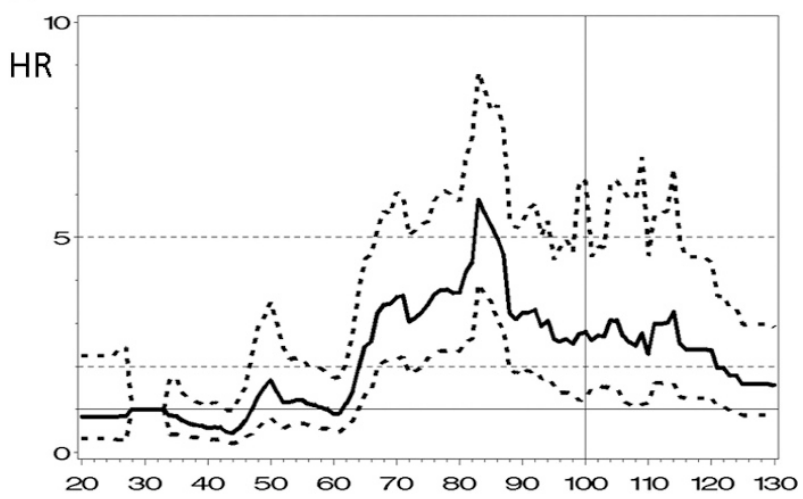

C

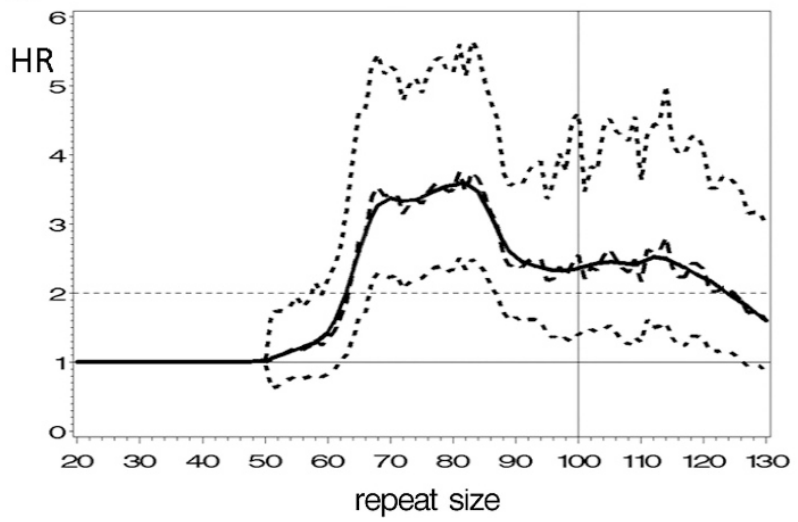

Fig. 2. Hazard ratios (HRs) for earlier menopause by repeat size of the largest allele compared with the referent category of 29-32 repeats. Panel A depicts HRs (solid line) for the Nijmegen study sample with 95\% confidence interval (dotted lines). Panel B depicts HRs (solid line) for the Atlanta study sample with $95 \%$ confidence interval (dotted lines). Panel C depicts the HRs for the total study sample (black dashed line) with $95 \%$ confidence interval (dotted lines) and superimposed a smoothed curve (solid line), using LOWESS smoother with smoothing factor 0.1 . The horizontal solid line depicts $H R=1$ and the two dashed lines depict $H R=2$ and $H R=5$. 
Table 2 Analyses of association of possible risk factors with age at menopause by Cox proportional hazards model with robust estimate for family relations

\begin{tabular}{|c|c|c|c|c|c|c|c|c|c|c|c|c|}
\hline \multirow[b]{3}{*}{ Factors } & \multicolumn{6}{|c|}{ Premutation } & \multicolumn{6}{|c|}{ Noncarrier } \\
\hline & \multicolumn{2}{|c|}{ Univariate } & \multicolumn{2}{|c|}{ Multivariate } & \multicolumn{2}{|c|}{ Final model } & \multicolumn{2}{|c|}{ Univariate } & \multicolumn{2}{|c|}{ Multivariate } & \multicolumn{2}{|c|}{ Final model } \\
\hline & HR & $P$ & HR & $P$ & HR & $P$ & HR & $P$ & HR & $P$ & HR & $P$ \\
\hline \multicolumn{13}{|l|}{ Genetic } \\
\hline$F M R 1$ : risk index by repeat & 1.26 & $<0.001^{a}$ & 1.43 & $<0.001^{a}$ & 1.63 & $<0.001^{a}$ & - & - & - & - & 1 & NA \\
\hline $\begin{array}{l}\text { Mean menopausal age } 1 \mathrm{st}^{a} \\
\text { degree relatives }\end{array}$ & 0.97 & $0.02^{a}$ & & & 0.98 & 0.24 & 0.90 & $0.007^{a}$ & 0.91 & $0.01^{a}$ & 0.91 & $0.01^{a}$ \\
\hline \multicolumn{13}{|l|}{ Environmental } \\
\hline Smoking (ever) & $1.30^{b}$ & $0.048^{a}$ & 1.34 & $0.02^{a}$ & $1.35^{b}$ & $0.02^{a}$ & $1.44^{b}$ & $0.03^{a}$ & 1.46 & $0.03^{a}$ & 1.46 & $0.03^{a}$ \\
\hline BMI & $0.98^{b}$ & 0.056 & & & & & $1.00^{b}$ & 0.87 & & & & \\
\hline \multicolumn{13}{|l|}{ Reproductive } \\
\hline Menarche & $1.04^{b}$ & 0.41 & & & & & $1.05^{b}$ & 0.3054 & & & & \\
\hline Parity & $0.85^{b}$ & 0.25 & & & & & $0.92^{b}$ & 0.5673 & & & & \\
\hline Hormone use (ever) & $1.14^{b}$ & 0.34 & & & & & $0.99^{b}$ & 0.9272 & & & & \\
\hline \multicolumn{13}{|l|}{ Confounders } \\
\hline Age at interview & 1.02 & $0.003^{a}$ & & & & & 1.02 & $0.01^{a}$ & & & & \\
\hline Ascertainment site & 1.52 & $0.004^{a}$ & 1.92 & $<0.001^{a}$ & 1.77 & $<0.001^{a}$ & 1.93 & $<0.001^{a}$ & 1.99 & $<0.001^{a}$ & 1.99 & $<0.001^{a}$ \\
\hline
\end{tabular}

${ }^{a}$ Significant at level $\alpha=0.05$.

${ }^{b}$ All environmental and reproductive factors were adjusted for the FMRI risk group at univariate analysis. Multivariate, only significantly associated factors are shown. Final model shows all variables selected for our model based on significant association for either premutation model or noncarrier model to improve comparability. HR $>1$ indicates increased odds for an earlier menopause (risk factor), and $\mathrm{HR}<1$ indicates a longer time to menopause (protective factor).

$\mathrm{HR}$, hazard ratio; FMR1, fragile $\mathrm{X}$ mental retardation 1 .

continuous variables were of the correct functional form. For premutation carriers, the RIR was highly associated with age at menopause, with an HR of 1.26 for univariate analysis and 1.43 for multivariate analysis (Table 2). By univariate analysis, two other potential predictors showed a significant association, i.e., mean menopausal age of first-degree relatives with a premutation $(\mathrm{HR}=0.97)$ and smoking $(\mathrm{HR}=1.30)$. Among noncarriers, except for RIR, the same putative predictors were univariate significantly associated with menopausal age: $\mathrm{HR}=0.90$ for the effect of mean menopausal age of first-degree relatives and $\mathrm{HR}=1.44$ for the effect of smoking. The HR $<1$ for mean age at menopause among first-degree relatives suggests a protective factor or one that helps to increase the age at menopause. The HR $>1$ for smoking indicates a risk factor or one that reduces the age at menopause. Two possible confounders, age at interview and ascertainment site, were significantly associated with menopausal age in both groups, although only ascertainment site held significance at multivariate levels.

\section{A FXPOI prediction model}

A model to generate risk estimates for FXPOI, or menopausal age before age 40 years, for premutation carriers was built on the significant predictors based on multivariate analysis by backward selection of the predictors with significant association for univariate analysis, i.e., RIR and smoking as a predictor for menopausal age in premutation carriers and ascertainment site (Table 2). For comparison, a model for noncarriers was developed in the same way and included mean menopausal age of first-degree relatives, smoking, and ascertainment site. We decided to also include mean age at menopause of first- degree relatives into the prediction model for the premutation carriers because of our findings on heritability (description of our heritability analyses and results are available as Supplemental Digital Content 1, http://links.lww.com/GIM/A174) and because we saw an additive genetic effect in our previous study. ${ }^{24}$ These models predicted the probability of becoming postmenopausal before 40 years of age with an accuracy of \pm 0.10 among premutation carriers and \pm 0.07 for noncarriers.

The high-risk profile for premutation carriers consisted of a repeat size window centered on 80 repeats, smoker, ascertainment site Nijmegen, and young mean menopausal age of firstdegree family members, whereas the low-risk profile was based on the noncarrier model and consisted of a repeat size 50, never smoked, ascertainment site Atlanta, and late mean menopausal age of first-degree relatives, respectively. According to this model, women with this high-risk profile have 38\% (95\% CI: 29-47) chance of being menopausal before the age of 40 years and, thus, are at high risk for FXPOI (Fig. 3). The effect of ascertainment location is a 3-4 years earlier menopause for the Nijmegen cohort as shown for different repeat sizes in Figure 4. On average, the fit of the premutation carrier and the noncarrier model was good, as shown by inspecting the tertile plots (Fig. 5). Only for subjects in the tertiles with the latest predicted menopause, the observed Kaplan-Meier curves suggested a somewhat later start of menopause as predicted by the Cox model, but this difference was only a few months. Internal validation of the premutation model showed that the optimism in $R^{2}$ Nagelkerke, original value 0.09 (95\% CI: $\left.0.04-0.15\right)$, was 0.01 . The optimism in the concordance index $c$, original value 0.66 (95\% CI: 0.61-0.70), was 0.008. The linear shrinkage 


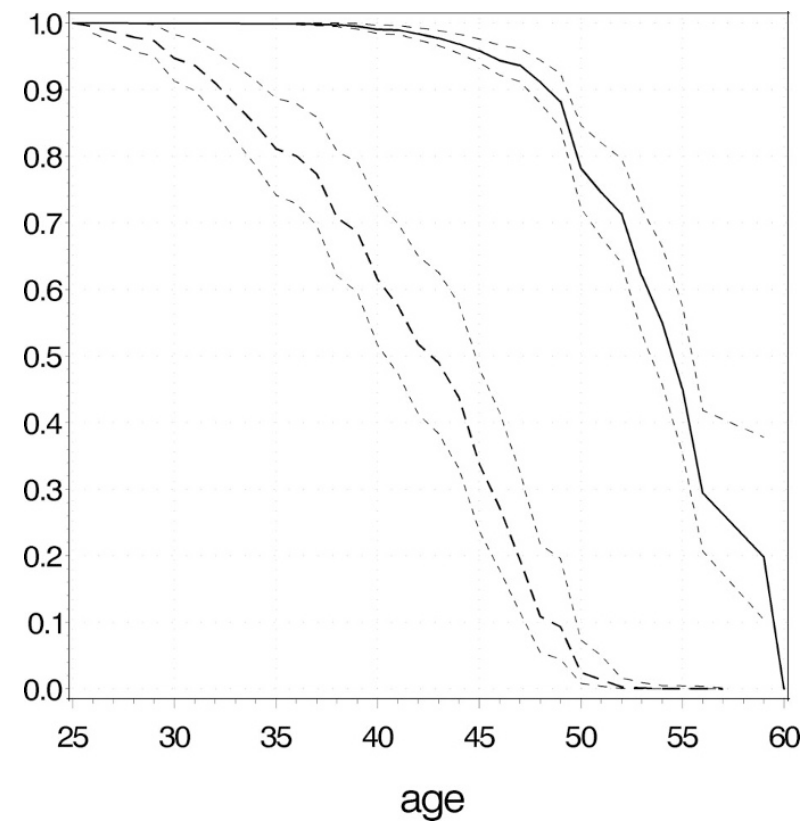

Fig. 3. Predicted survival curves for reaching postmenopausal state for high-risk premutation women (with repeat size 80 , smoking, mean menopausal age of first-degree relatives 38 years, and ascertainment location Nijmegen; dashed curve) and low risk, based on the noncarriers model (repeat size 50, never smoked, mean menopausal age of first-degree relatives 55, and location Atlanta; solid curve) with $95 \%$ confidence bands.

factor seemed to be 0.95 . For the noncarriers, comparable values were seen: $R^{2}{ }_{\text {Nagelkerke }}=0.09$ (95\% CI: $\left.0.06-0.18\right)$, optimism $=0.02 ; c=0.69$ (95\% CI: $0.62-0.75)$, optimism $=$ 0.005 , and shrinkage factor $=0.90$.

\section{DISCUSSION}

By using a selected cohort of 1068 women, we built a FXPOI prediction model based on FMRI CGG repeat size, menopausal age of first-degree relatives, and the environmental factor smoking, together with a correction for ascertainment location. We found that this model can predict the risk for menopause at a certain age, as indicated by the acceptable fit, the small CIs, and the good results of the internal validation procedure (i.e., small amounts of optimism in $R^{2}$ and concordance index $c$ and a shrinkage factor close to 1). To our knowledge, this is the first model developed to help predict menopausal age in FMRI premutation carriers. Other reproductive factors such as menarche and hormone use were not significantly associated with menopausal age in the premutation carriers and, thus, not included in the prediction model. Van Noord et al. ${ }^{12}$ reported that the impact of environmental factors on menopausal age is limited, which seems to be true for premutation carriers as well. A prominent association with smoking (HR of 1.34) and menopausal age in premutation carriers was found, in accordance with data previously reported by Allen et al. ${ }^{13}$ The toxic effect of smoking could give rise to destruction of the primordial oocytes at the level of the ovary ${ }^{28}$ and could, therefore, accelerate the onset of FXPOI.

Many studies dealing with relationship between various factors, such as menarche, OC use, BMI, and parity, and early

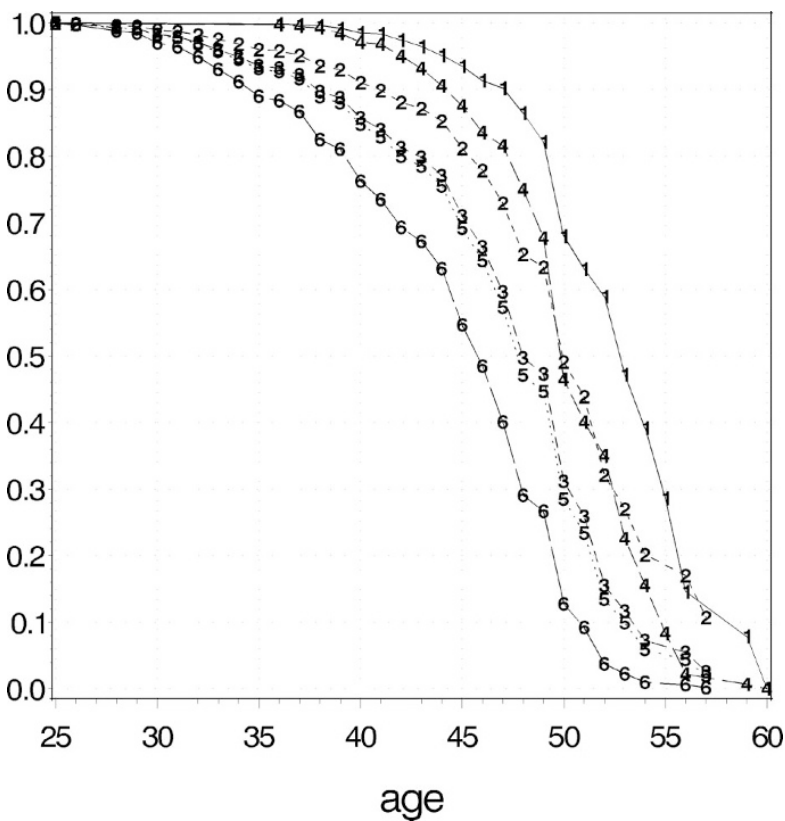

Fig. 4. To illustrate the effect of ascertainment location, Kaplan-Meier estimates of probability of being postmenopausal are provided for each ascertainment location varying repeat size and mean age of menopause for firstdegree relatives and smoking. For the Atlanta location: repeat size 50, smoking, mean menopausal age of firstdegree noncarrier relatives (curve 1); repeat size 70, smoking, mean menopausal age of first-degree relatives carrying a premutation (curve 2); and repeat size 80, smoking, mean menopausal age of first-degree relatives carrying a premutation (curve 3). For the Nijmegen location: repeat size 50, smoking, mean menopausal age of first-degree noncarrier relatives (curve 4); repeat size 70, smoking, mean menopausal age of first-degree relatives carrying a premutation (curve 5); and repeat size 80, smoking, mean menopausal age of first-degree relatives carrying a premutation (curve 6). The curves 1 and 4 are based on the noncarrier model and the curves 2, 3, 5, and 6 on the premutation model.

menopause (including POI and early menopause), have turned out to be controversial. ${ }^{12,29-32}$ In our set of premutation carriers, no significant association with menopausal age was seen for these factors.

In the final model, the FMR1 CGG repeat size, represented by "risk index by repeat size (RIR)," is the most influential predictor. This was expected as the repeat size has been strongly associated with FXPOI before. ${ }^{13-16}$ To accommodate the nonlinear association of repeat size and risk for FXPOI and to avoid the somewhat arbitrary classification of repeat groups, we used an HR-based approach and incorporated these risks relative to a reference repeat group into the prediction model. In this way, the fast rise in risk after $60 \mathrm{CGG}$ repeats and the slower decline in risk after $100 \mathrm{CGG}$ repeats can be modeled. The HR pattern for repeat size indicates that a significant high risk for FXPOI starts earlier and continues longer than what was previously reported. ${ }^{13,14}$ Because of the low HRs from 55 to 65 repeats, the higher risk at the end of the previously defined 55-79 premutation range might not have been evident before. The high-risk approximately 105-110 repeats has never been described before 

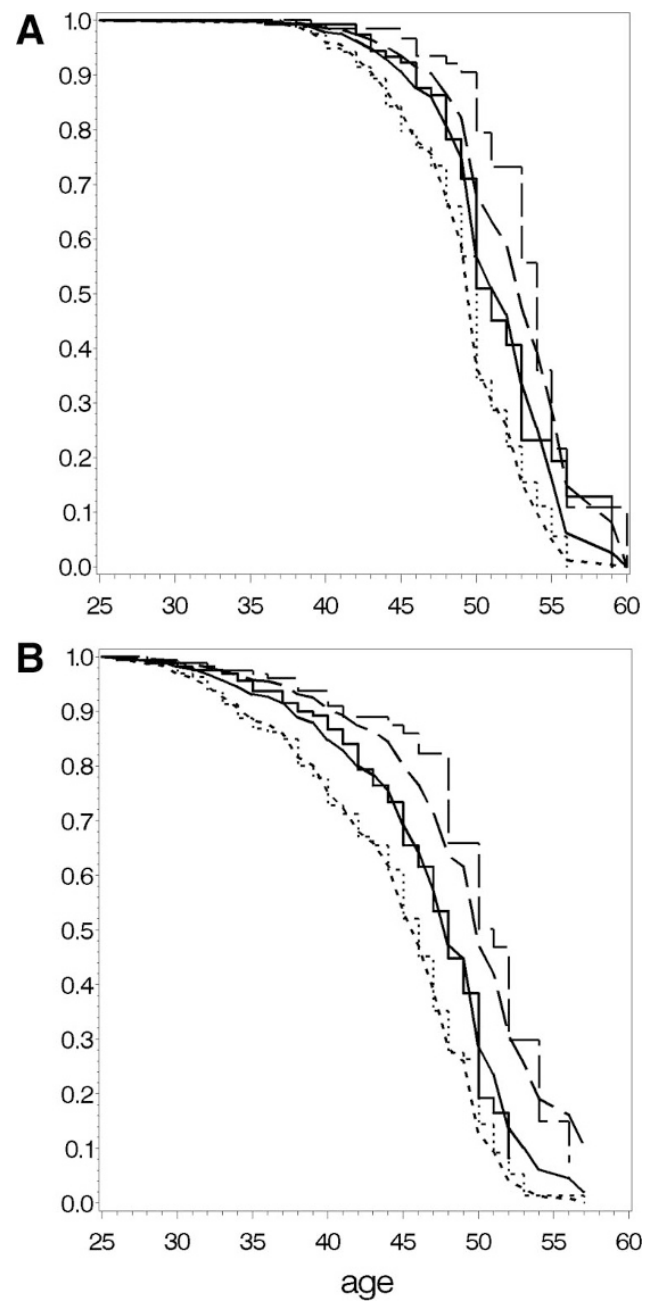

Fig. 5. Fit of our prediction models. Panel A shows Kaplan-Meier estimates of observed menopausal age and the predicted menopause by our model for noncarriers for tertiles based on observed menopausal age. Panel B shows Kaplan-Meier estimates of observed menopausal age and the predicted menopause by our model for premutation carriers for tertiles. Smoothed curves represent the observed data and stepped curves the predicted data.

and may be attributed to small numbers (three women with a repeat size of 110 and one with a repeat size of 105 and a menopause at a relatively young age). An isolated high risk for FXPOI based on a few repeats is considered unlikely. Despite a small sample size, a significant risk was also seen for 160 repeats. Thus, an elevated risk above 130 repeats could not be excluded and requires further assessment.

Discrepancies exist about risk for ovarian insufficiency among intermediate CGG repeat size carriers. Our analysis confirms that intermediate sized FMRI CGG repeats should not be considered a high-risk factor for ovarian insufficiency, as recently reported by Bennett et al. ${ }^{33}$ In addition, we did not observe a higher risk for menopause at an early age among intermediate repeat alleles. We examined this association using the defined genotypes by Gleicher et al., ${ }^{34,35}$ where they defined a normal allele as those with $26-34$ repeats and abnormal alleles as those outside of this range. None of the women in our study who were either heterozygous or homozygous abnormal had menopausal age before the age of 40 years. Most had menopausal age above 45 years. Additional studies need to be conducted to further evaluate the association of these genotypes on phenotypes associated with ovarian function. ${ }^{36-39}$

We observed a significant effect of ascertainment location of our study population. Several possible explanations for this effect have been explored. We excluded a difference in distribution of CGG repeat sizes and the proportion of premutation carriers in both groups as a basis for the observed ascertainment location effect. Also, a difference in genetic background of the Dutch and American populations seems unlikely because of the inclusion of whites only. Most white Americans are from $\mathrm{Eu}-$ ropean descent. One explanation could be the manner in which the interviews were taken. In the Atlanta study, the reproductive questionnaire was part of a large assessment, often including neuropsychological, medical, and neurologic assessments as well. The Nijmegen study was set up for reproductive evaluation and the study information included a description of FXPOI, which could result in participants reporting menopause at an earlier age than when it actually occurred.

A limitation of all cross-sectional studies is the use of timedependent variables such as smoking, BMI, parity, and hormone use. Such variables are preferably analyzed by an extended Cox proportional hazards model. However, exact information of these variables per subject at different time points between 25 years of age and the start of menopause was lacking. Therefore, these variables were applied as if they were fixed at the age of 25 years. Even though this is a raw approximation of the reality, results based on this approach are at least indicative for the overall effect of these variables on menopausal age. In addition, age at menopause is a transitory state and subject to reporting and recall bias. A previous study on recall of menopausal age showed a bias toward the mean as women deviated from the final menstrual period. ${ }^{40}$ In our case, recall and memory bias might have led to an earlier menopausal age because of awareness for FXPOI.

Furthermore, we have used age at menopause as a surrogate for severity of FXPOI, but in general, POI should not be considered a natural menopause, because of intermittent ovarian function and a $5-10 \%$ chance of pregnancy after diagnosis. ${ }^{41}$ We used the definition of amenorrhea for at least 1 year due to menopause as a uniform criterion that applies to older and younger women.

Taken together, our data strongly indicate that a prediction model for FXPOI is feasible, when taking ascertainment site into account. This effect clearly confirms the importance of data collection and ascertainment protocols, as they could bias results. Nevertheless, we consider our model as a first step in developing clinically applicable risk estimates for FXPOI, which could facilitate counseling. To further develop and individualize risk estimates for FXPOI, a predictor based on endocrine markers, such as anti-Müllerian hormone, could be included. Anti-Müllerian hormone is an early marker of ovarian reserve and a very promising predictor for menopause and POI in general, and for premutation carriers in particular. ${ }^{42}$ The decline in ovarian reserve with time in premutation carriers with FXPOI, however, is not fully comprehended yet. Development of reference values of anti-Müllerian hormone by age for premutation carriers might allow early identification of women at high risk for FXPOI. In addition, examination of different ethnic/racial groups might be important in these prediction models. Studies have shown that the frequency distribution of FMR1 alleles differs among groups ${ }^{43,44}$; however, no studies have examined whether the effect of the FMR1 alleles differs by ethnic/racial group. Using our current model based on RIR, 
mean menopausal age of first-degree relatives with the same mutation status and smoking, premutation carriers with a high risk for FXPOI might benefit from monitoring of ovarian reserve regularly and consider vitrification, i.e., cryopreservation of oocytes.

\section{ACKNOWLEDGMENTS}

This work was supported, in part, by National Institutes of Health Grants NIH RO1 HD29909 and NIH PO1 HD35576 (S.L.S. and E.G.A.). The authors thank the volunteers and their families whose participation made the work possible.

\section{REFERENCES}

1. Cronister A, Schreiner R, Wittenberger M, Amiri K, Harris K, Hagerman RJ. Heterozygous fragile $\mathrm{X}$ female: historical, physical, cognitive, and cytogenetic features. Am J Med Genet 1991;38:269-274.

2. Schwartz CE, Dean J, Howard-Peebles PN, et al. Obstetrical and gynecological complications in fragile $\mathrm{X}$ carriers: a multicenter study. Am J Med Genet 1994;51:400-402.

3. Allingham-Hawkins DJ, Babul-Hirji R, Chitayat D, et al. Fragile X premutation is a significant risk factor for premature ovarian failure: the International Collaborative POF in fragile X study-preliminary data. Am J Med Genet 1999;83:322-325.

4. Murray A, Webb J, Grimley S, Conway G, Jacobs P. Studies of FRAXA and FRAXE in women with premature ovarian failure. $J$ Med Genet 1998;35: 637-640.

5. Welt CK. Primary ovarian insufficiency: a more accurate term for premature ovarian failure. Clin Endocrinol (Oxf) 2008;68:499-509.

6. Verkerk AJ, Pieretti M, Sutcliffe JS, et al. Identification of a gene (FMR-1) containing a CGG repeat coincident with a breakpoint cluster region exhibiting length variation in fragile X syndrome. Cell 1991;65:905-914.

7. Kronquist KE, Sherman SL, Spector EB. Clinical significance of tri-nucleotide repeats in fragile $\mathrm{X}$ testing: a clarification of American College of Medical Genetics guidelines. Genet Med 2008;10:845-847.

8. te Velde ER, Pearson PL. The variability of female reproductive ageing. Hum Reprod Update 2002;8:141-154.

9. Cramer DW, Xu H, Harlow BL. Family history as a predictor of early menopause. Fertil Steril 1995;64:740-745.

10. de Bruin JP, Bovenhuis H, van Noord PA, et al. The role of genetic factors in age at natural menopause. Hum Reprod 2001;16:2014-2018.

11. Treloar SA, Do KA, Martin NG. Genetic influences on the age at menopause. Lancet 1998;352:1084-1085.

12. van Noord PA, Dubas JS, Dorland M, Boersma H, te Velde ER. Age at natural menopause in a population-based screening cohort: the role of menarche, fecundity, and lifestyle factors. Fertil Steril 1997;68:95102

13. Allen EG, Sullivan AK, Marcus M, et al. Examination of reproductive aging milestones among women who carry the FMR1 premutation. Hum Reprod 2007;22:2142-2152.

14. Sullivan AK, Marcus M, Epstein MP, et al. Association of FMR1 repeat size with ovarian dysfunction. Hum Reprod 2005;20:402-412.

15. Ennis S, Ward D, Murray A. Nonlinear association between CGG repeat number and age of menopause in FMR1 premutation carriers. Eur J Hum Genet 2006;14:253-255.

16. Tejada MI, Garcia-Alegria E, Bilbao A, et al. Analysis of the molecular parameters that could predict the risk of manifesting premature ovarian failure in female premutation carriers of fragile X syndrome. Menopause 2008; 15:945-949.

17. Nolin SL, Brown WT, Glicksman A, et al. Expansion of the fragile X CGG repeat in females with premutation or intermediate alleles. Am J Hum Genet $2003 ; 72: 454-464$
18. Fu YH, Kuhl DP, Pizzuti A, et al. Variation of the CGG repeat at the fragile $\mathrm{X}$ site results in genetic instability: resolution of the Sherman paradox. Cell 1991;67:1047-1058.

19. Zeger SL, Liang KY. Longitudinal data analysis for discrete and continuous outcomes. Biometrics 1986;42:121-130.

20. Brown WT, Houck GE Jr, Jeziorowska A, et al. Rapid fragile X carrier screening and prenatal diagnosis using a nonradioactive PCR test. JAMA 1993;270:1569-1575.

21. Cleveland WS. Robust locally weighted regression and smoothing scatterplots. J Am Stat Assoc 1979;74:829-836.

22. Do KA, Treloar SA, Pandeya N, et al. Predictive factors of age at menopause in a large Australian twin study. Hum Biol 1998;70:1073-1091.

23. Torgerson DJ, Avenell A, Russell IT, Reid DM. Factors associated with onset of menopause in women aged 45-49. Maturitas 1994;19:83-92.

24. Hunter JE, Epstein MP, Tinker SW, Charen KH, Sherman SL. Fragile $\mathrm{X}$-associated primary ovarian insufficiency: evidence for additional genetic contributions to severity. Genet Epidemiol 2008;32:553-559.

25. Grambsch PM, Therneau TM. Proportional hazards tests and diagnostics based on weighted residuals. Biometrika 1994;81:515-526.

26. Therneau TM, Grambsch PM, Fleming TR. Martingale-based residuals for survival models. Biometrika 1990;77:147-160.

27. Efron B, Tibshirani R. An introduction to the bootstrap. New York: Chapman and Hall, 1993.

28. Neal MS, Zhu J, Foster WG. Quantification of benzo[a]pyrene and other PAHs in the serum and follicular fluid of smokers versus non-smokers. Reprod Toxicol 2008;25:100-106.

29. Progetto Menopausa Italia Study Group. Premature ovarian failure: frequency and risk factors among women attending a network of menopause clinics in Italy. BJOG 2003;110:59-63.

30. Cramer DW, Xu H, Harlow BL. Does “incessant" ovulation increase risk for early menopause? Am J Obstet Gynecol 1995;172:568-573.

31. de Vries E, den Tonkelaar I, van Noord PA, van der Schouw YT, te Velde ER, Peeters PH. Oral contraceptive use in relation to age at menopause in the DOM cohort. Hum Reprod 2001;16:1657-1662.

32. Stanford JL, Hartge P, Brinton LA, Hoover RN, Brookmeyer R. Factors influencing the age at natural menopause. J Chronic Dis 1987;40:995-1002.

33. Bennett CE, Conway GS, Macpherson JN, Jacobs PA, Murray A. Intermediate sized CGG repeats are not a common cause of idiopathic premature ovarian failure. Hum Reprod 2010;25:1335-1338.

34. Gleicher N, Weghofer A, Barad DH. Ovarian reserve determinations suggest new function of FMR1 (fragile $\mathrm{X}$ gene) in regulating ovarian ageing. Reprod Biomed Online 2010;20:768-775.

35. Gleicher N, Barad DH. The FMR1 gene as regulator of ovarian recruitment and ovarian reserve. Obstet Gynecol Surv 2010;65:523-530.

36. Bodega B, Bione S, Dalpra L, et al. Influence of intermediate and uninterrupted FMR1 CGG expansions in premature ovarian failure manifestation. Hum Reprod 2006;21:952-957.

37. Bretherick KL, Fluker MR, Robinson WP. FMR1 repeat sizes in the gray zone and high end of the normal range are associated with premature ovarian failure. Hum Genet 2005;117:376-382.

38. Gleicher N, Weghofer A, Barad DH. A pilot study of premature ovarian senescence: I. Correlation of triple CGG repeats on the FMR1 gene to ovarian reserve parameters FSH and anti-Mullerian hormone. Fertil Steril 2009;91:1700-1706

39. Gleicher N, Weghofer A, Oktay K, Barad D. Relevance of triple CGG repeats in the FMR1 gene to ovarian reserve. Reprod Biomed Online 2009;19:385-390.

40. Rockhill B, Colditz GA, Rosner B. Bias in breast cancer analyses due to error in age at menopause. Am J Epidemiol 2000;151:404-408.

41. Nelson LM, Covington SN, Rebar RW. An update: spontaneous premature ovarian failure is not an early menopause. Fertil Steril 2005;83:1327-1332.

42. Rohr J, Allen EG, Charen K, et al. Anti-Mullerian hormone indicates early ovarian decline in fragile $\mathrm{X}$ mental retardation (FMR1) premutation carriers: a preliminary study. Hum Reprod 2008;23:1220-1225.

43. Crawford DC, Acuna JM, Sherman SL. FMR1 and the fragile X syndrome: human genome epidemiology review. Genet Med 2001;3:359-371.

44. Gleicher N, Weghofer A, Barad DH. Effects of race/ethnicity on triple CGG counts in the FMR1 gene in infertile women and egg donors. Reprod Biomed Online 2010;20:485-491. 